\title{
Fast 3D Map Matching Localisation Algorithm
}

\author{
Miguel Pinto \\ INESC Porto - Institute for Systems and Computer Engineering of Porto \\ Faculty of Engineering, University of Porto, Porto, Portugal \\ Email: dee09013@fe.up.pt \\ A. Paulo Moreira, Aníbal Matos, Héber Sobreira, Filipe Santos \\ INESC Porto - Institute for Systems and Computer Engineering of Porto \\ Faculty of Engineering, University of Porto, Porto, Portugal \\ Email: \{amoreira,anibal,dee09025,dee09043\} @ fe.up.pt
}

\begin{abstract}
A new and fast methodology is discussed as a solution to pinpointing the location of a robot, in a robust way, without environment preparation, even in dynamic scenarios. This solution does not require a high computational power.

The methodology is a three-dimensional map based approach, which uses the 3D map of the surrounding environment and data acquired by a tilting Laser Range Finder (LRF), to pinpoint the robot pose. Experimental results about the accuracy of the proposed method are presented in this paper.
\end{abstract}

Index Terms - Robot localisation, 3D matching, EKF-SLAM, Laser Range Finder

\section{INTRODUCTION}

To be truly autonomous, a robot must be able to pinpoint their location inside dynamic environments, moving in an unlimited area, without preparation needs. In order to fulfil this definition of autonomy, the fundamental motivation and opportunity of this work, was the implementation of a robust strategy of localisation that runs online in a short execution time.

The developed approach is a three-dimensional map based localisation method, with the objective of solve the problem of accumulative error when the odometry is used, using the environment infrastructure, without constraints in terms of the navigation area and with no need of prepare the environment with artificial landmarks or beacons.

The localisation methodology is divided in the following steps: 1) pre-localisation and mapping, performed offline and only once, to obtain the map of an indoor environment; here it is intended to navigate with a vehicle equipped with an tilting LRF acquiring threedimensional data; and 2) localisation, which execute the vehicle self-localisation during its normal operation; this is used online using a matching algorithm and the obtained previous map.

It is applied to the RobVigil robot, a differential drive vehicle, shown in Figure 1 called RobVigil.

Manuscript accepted November 5, 2012.

Corresponding author: Miguel Pinto, email: dee09013@fe.up.pt
The RobVigil is equipped with sensors to detect dangerous situations, like fires, floods or gas leaks. It is equipped as well with three surveillance cameras. The main application of the RobVigil is the surveillance of public facilities, i.e. dynamic environments, allowing systematic and uninterruptable inspection routines, with minimum human intervention.

The RobVigil, performing a surveillance routine, in a shopping mall is shown in Erro! A origem da referência não foi encontrada..

The LRF - Hokuyo URG-04LX-UG01 - was used to perceive the environment. To obtain a three-dimensional sensor, a rotating platform was created based on the dc servo motor, the AX-12 Dynamixel Bioloid. The complete tilting LRF solution is shown in Figure 2. The tilting LRF has a distance range of 5 metres and acquires 769 points at every 100 milliseconds.
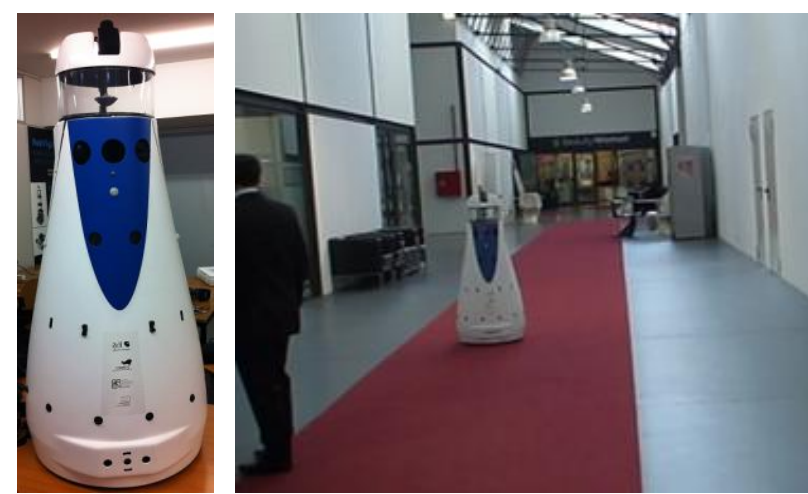

Figure 1 Left: the RobVigil robot equipped with the tilting LRF at top. Right: the robot performing a surveillance routine..

As the localisation algorithm is applied in the RobVigil, which navigates in public facilities, with people an dynamic objects crossing the navigation area, only data and the map about the headroom of the building (upper side-remains static during large periods of time), is used aiming to improve the methodology accuracy and robustness. Videos and downloads about this threedimensional map based methodology can be found at [1]. 


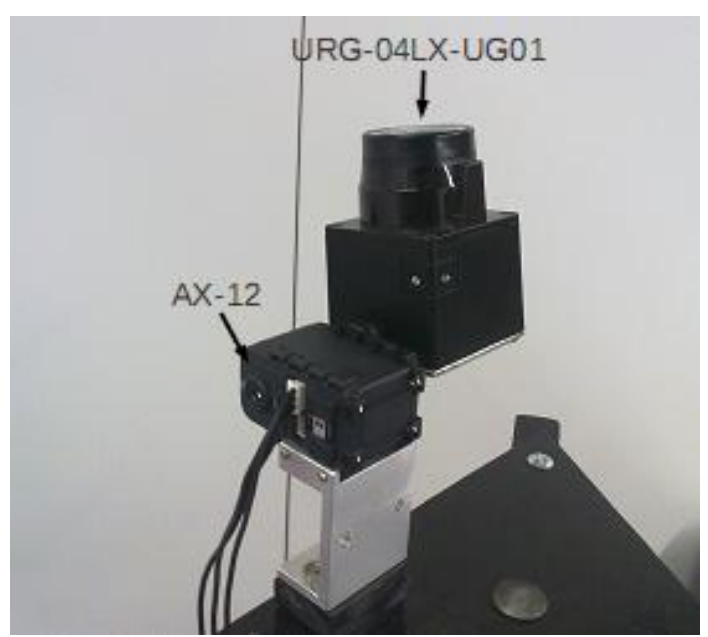

Figure 2 The tilting LRF developed.

\section{LITERATURE REVIEW}

Different sensors and techniques for the localisation of vehicles are described in [1]. These sensors and techniques are divided into absolute and relative localisation.

The dead-reckoning are sensors of relative localisation leading to an increase of the error over time. The most commonly used is the odometry.

Due to their high frequency rate, the dead-reckoning are commonly fused with more complex localisation techniques or sensors, through probabilistic methods as is example the Kalman Filters and the Particle Filters, [3].

Examples of sensors and techniques of absolute localisation are the attitude sensors, digital compasses, the GPS and passive or active beacons. The two essential localisation techniques based on active or passive beacons are: triangulation and trilateration, [4]. Unfortunately, this methods require environment preparation.

The algorithms concerning the localisation of mobile robots can be divided in two large areas: the matching and the Simultaneous Localisation and Mapping (SLAM) algorithms.

There are matching algorithms that need a prior knowledge about the navigation area, as is example [5]. Another example of a matching algorithm is the Perfect Match described by M. Lauren et al. [6], used in the Robotic Soccer Middle Size League (MSL) at RoboCup. The Perfect Match is a time saver algorithm.

There are other types of matching algorithms, which compute the overlapping zone between consecutive observations to obtain the vehicle displacement, [7].

The most common SLAM's solutions are: the Extended Kalman Filter (EKF-SLAM) [8], and the FastSlam/Rao-Blackwellized Particle Filters [9].

The EKF-SLAM computationally complexity is $\mathrm{O}\left(\mathrm{N}^{2}\right)$, while the FastSlam has a lower computational complexity, $\mathrm{O}(\mathrm{M} \log \mathrm{N})$, with $\mathrm{M}$ particles and where $\mathrm{N}$ landmarks.

\section{PRE-LOCALISATION \& MAPPING}

This phase is performed offline and only once, aiming to obtain the 3D occupancy grid of the building where it is intended the RobVigil navigation.

The obtained 3D occupancy grid is used before, during the vehicle normal operation, to pinpoint its self-location, in a fast way and on-line.

This phase has two crucial steps, the pre-localisation and mapping. The pre-localisation implements a SLAM solution. Once the SLAM has been applied, the 2D feature map with linear segments and points is obtained. The same map is after used to determine the vehicle's location (pre-localisation). The SLAM solution is based in the state of art EKF-SLAM algorithms, as the described in [3].

Finally, still offline and with the vehicle's position obtained, the three-dimensional map can be built and the respective distance and gradient matrices can be created and stored (mapping procedure)

To create the distance matrix, the distance transform is applied in the 3D space, on the occupancy grid of the building. Furthermore, the Sobel filter, again in the 3D space, is applied to obtain the gradient matrices, in both the directions $\mathrm{x}$ and $\mathrm{y}$. The stored distance and gradient matrices are used as look-up tables for the localisation procedure, in the normal vehicle operation, as described in the next section.

\section{LOCALISATION}

The light computational Perfect Match algorithm described in [6] by M.Lauren et al., was adapted from 2D to the 3D space, using Laser Range Finder data, maintaining the low computational requirements.

Therefore, the developed 3D Perfect Match is based on the steps: 1) matching error; 2) optimisation routine Resilient Back-Propagation (RPROP); and 3) second derivative.

The distance matrix, stored in memory is used to compute the matching error. The gradient matrices also stored in memory, are used to find convergence direction of the RPROP algorithm and compute the resulting covariance estimation.

The RPROP algorithm takes the previous state of the vehicle $X_{\text {Match }}(n-1)$ and estimates a new vehicle position $X_{\text {Match }}(n)$, which is used in the next iteration.

Consider now that the list of points of a Laser Range Finder scan, in the world frame, is PntList(i) = $\left(\mathrm{x}_{\mathrm{i}}, \mathrm{y}_{\mathrm{i}}, \mathrm{z}_{\mathrm{i}}\right)^{\mathrm{T}}$. The cost value is given by:

$$
E=\sum_{i=1}^{\text {PntList.Count }} E_{i}, \quad E_{i}=1-\frac{L_{c}^{2}}{L_{c}^{2}+d_{i}^{2}}
$$

in which, $d_{i}$ and $E_{i}$ represent the distance matrix and cost function values.

The parameter $L_{c}$ is used to negligect points with and error $E_{i}$, higher than itself. The used value was 1 meter. The resulting state $\mathrm{X}_{\text {Match }}$ is given by the RPROP 
algorithm. The match $\mathrm{P}_{\text {Match }}$ covariance is given by the following matrix:

$$
P_{\text {Match }}=\operatorname{diag}\left(K_{X Y} / \frac{\partial^{2} E}{\partial x_{v}^{2}}, K_{X Y} / \frac{\partial^{2} E}{\partial y_{v}^{2}}, K_{\theta} / \frac{\partial^{2} E}{\partial \theta_{v}^{2}}\right)
$$

in which, $\operatorname{diag}(., \ldots)$ is the diagonal matrix $3 \times 3$.

The parameters in the equation $2, \mathrm{~K}_{\mathrm{XY}}$ and $\mathrm{K}_{\theta}$, are normalized values. The algorithm was tested with values of $K_{X Y}$ and $K_{\theta}$ in the gap between $\left[10^{-6}, 10^{-1}\right]$. The best performance achieved was $\mathrm{K}_{\mathrm{XY}}=10^{-3}$ and $\mathrm{K}_{\theta}=10^{-3}$. To more details about the Perfect Match algorithm see [6].

The localisation procedure uses the result of the 3D Perfect Match in a position tracking algorithm, which has three fundamental stages: 1) the Kalman Filter Prediction, 2) the 3D Perfect Match procedure, and 3) the Kalman Filter Update. The Kalman Filter equations can be seen in more detail in [3].

The Kalman filter prediction stage takes the previously estimated vehicle state $\mathrm{X}_{\mathrm{v}}$ and, using odometry, it estimates the next vehicle state $\mathrm{X}_{\text {Odo }}$.

The 3D Perfect Match algorithm takes the state $\mathrm{X}_{\text {Odo }}$ given by the Kalman Filter Prediction, and outputs the resulting state $\mathrm{X}_{\text {Match }}$ and covariance $\mathrm{P}_{\text {Match }}$.

The Kalman Filter Update stage combines the estimated states $\mathrm{X}_{\text {odo }}$ and the $\mathrm{X}_{\text {Match }}$ using the EKF Update equations, [3].

\section{EXPERIMENTAL RESULTS}

The Figure 1 shows the architects' plant about a building on the Faculty of Engineering of the University Porto, in Portugal. The solid black colour on the Figure 1, shows the zone where the pre-localisation \& mapping, described on section III, was applied becoming possible to obtain the 3D occupancy grid used during the localisation, described on section IV.

The map was obtained with success. The Figure 4 and Figure 5, with two different perspectives of view, shows the $3 \mathrm{D}$ occupancy grid of this large indoor environment.

In the Figure 4 and Figure 5, the red points represent the upper side of the building (above 1.8 metres of height), almost static, used to perform the 3D matching.

Aiming to evaluate the accuracy of the localisation algorithm based on the 3D matching presented in this paper, experiments were made with the high precision Ground Truth (GND) positioning system, the SICK Nav350, a commercial solution for autonomous guided vehicles (AGV).

The SICK Nav350 uses reflectors to output its self location, with a sample rate of 125 milliseconds and an accuracy of 4 millimetres to 25 millimetres.

The Figure 6 shows the trajectory in a corridor of the building, whose map is shown in Figure 4 and Figure 5. Only in this part of the building it is available the GND system. Therefore, only in this corridor are shown accuracy results.

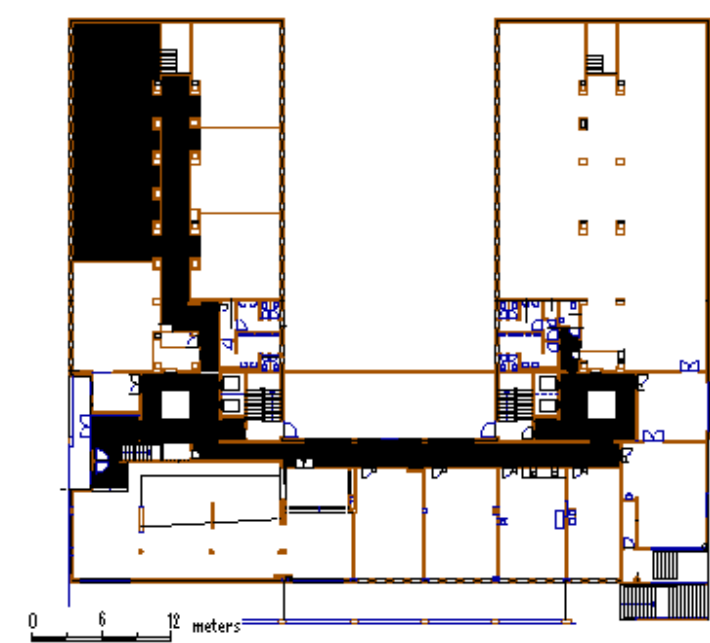

Figure 3 Architects' plant of the mapped environment.

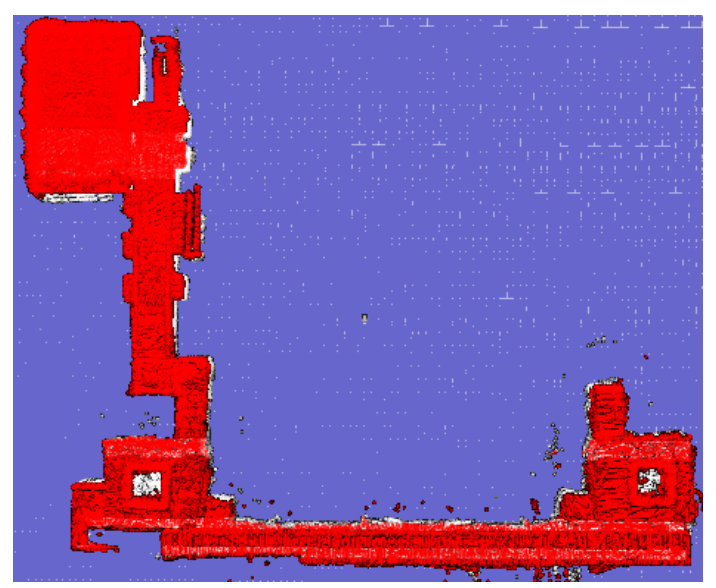

Figure 4 Occupancy grid of the scenario, with square shape of 60x60 metres.

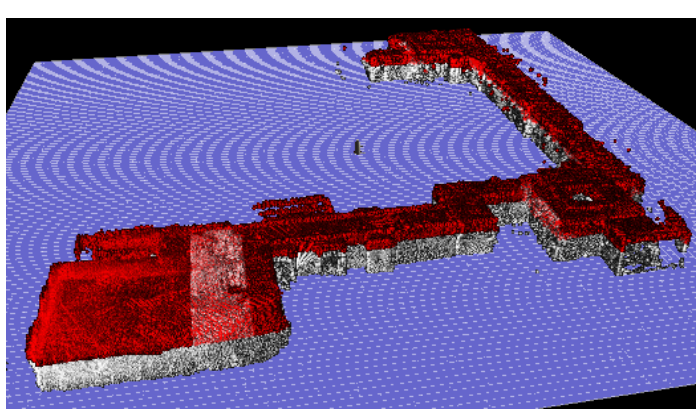

Figure 5 Occupancy grid of the scenario. Other perspective of view.

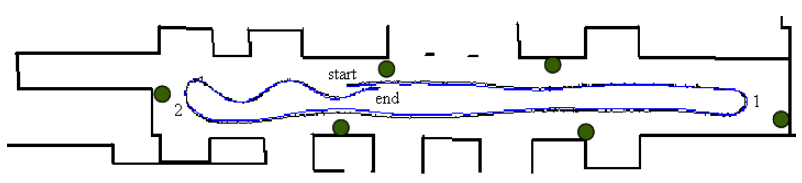

Figure 6 SICK Nav350 Vs estimated location. The corridor has 25 x 8 metres.

In the figure (Figure 6), the black line is the trajectory obtained with the Nav350, while the blue line is the 
trajectory estimated by the localisation algorithm (3D matching).

The Figure 7 shows the architects' plant about another building, in the Faculty of Engineering of the University Porto, in Portugal, where the methodology described in this paper was applied, aiming the vehicle's normal operation in this building. The solid black colour shows the zone where the methodology was applied.

The 3D occupancy grid about this building, obtained after applied the pre-localisation \& mapping, described on section III, used in the vehicle normal operation, aiming its localisation, as described on section IV, is shown in Figure 8 and Figure 9.

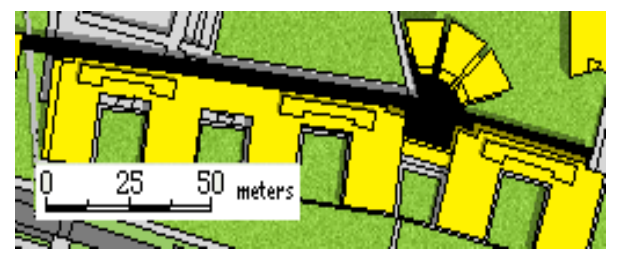

Figure 7 Architects' plant of the mapped environment.

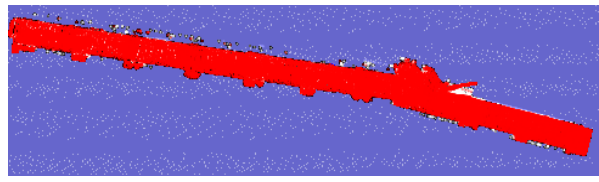

Figure 8 Occupancy grid of the scenario, with square shape of $60 \times 60$ metres.

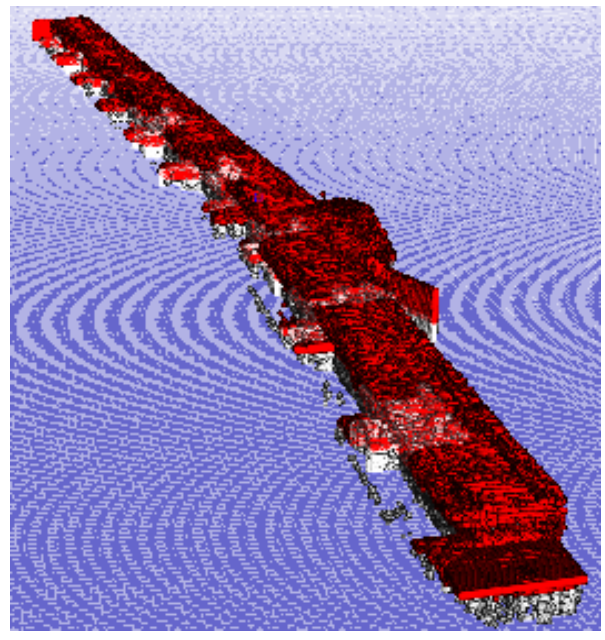

Figure 9 Occupancy grid of the scenario. Other perspective of view.

In this scenario, also the red points represent the upper side of the building (above 1.8 metres of height), almost static, used to perform the 3D matching.

Again, in this scenario the ground truth sensor (Sick Nav350) is available to perform the characterization of the accuracy of the proposed 3D Matching method. The Figure 10 shows the trajectory in a part of the building, whose map is shown in Figure 8 and Figure 9.

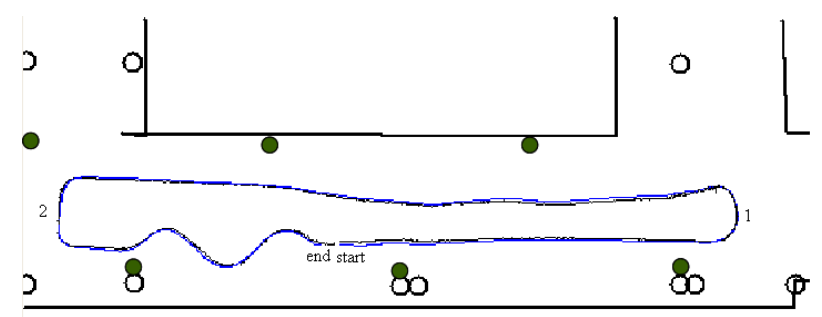

Figure 10 SICK Nav350 Vs estimated location. The corridor has 25 x 8 metres.

In the figure, the black line is the ground truth trajectory, while the blue line is the trajectory estimated by the localisation algorithm (3D matching).

In the experiments of Figure 6 and Figure 10, the average of the Euclidian distance error between the 3D matching and the Nav350 position is 0.08 metres, with a standard deviation of 0.086 metres. The absolute average orientation difference is $1.18^{\circ}$, with a standard deviation of $1.72^{\circ}$.

This experiments helped to realize that the localisation proposed here is sufficient accurate to be used in the surveillance task of the RobVigil robot.

The RobVigil moved in these experiments at an average speed of $0.4 \mathrm{~m} / \mathrm{s}$. The localisation algorithm takes an average execution time of 12 milliseconds, with 769 points of the tilting LRF in a Mini ITX, EPIA $\mathrm{M} 10000 \mathrm{G}$ with a $1.0 \mathrm{GHz}$ processor. In this experiment the tilting LRF rotates at a speed of $10 \mathrm{rpm}$.

The ICP algorithm described in [7] takes an average time of 76 milliseconds (can be higher) to find the optimal solution, using only 361 Laser Range Finder points, in a cycle of 200 milliseconds, in a Pentium IV $1.8 \mathrm{GHz}$.

\section{CONCLUSIONS}

The contributes made in this work were: adaptation of a light computational matching algorithm, the Perfect Match, to be used in the 3D space instead 2D, using Laser Range Finder data, maintaining the low computational requirements. Improvement of the fusion system between the matching algorithm described in [6] by M.Lauren et $a l$. and odometry data, using an EKF. Only 3D data about the upper side of a building (almost a static scenario) is used, becoming the localisation more robust and reliable, since in dynamic scenarios. The localisation methodology can be used with any observation module, which acquires 3D data: Kinect, 3D camera (MESA), stereo vision or commercial 3D LRF. Development of a localisation methodology that becomes the robot RobVigil an economically practicable robotic platform.

The three-dimensional map based localisation algorithm presented here, improves computational requirement comparatively to $2 \mathrm{D}$ and $3 \mathrm{D}$ Simultaneous Localisation and Mapping (SLAM) algorithms. Furthermore, the time necessary to locate the robot is also reduced comparatively to ICP algorithms. 


\section{ACKNOWLEDGEMENTS}

This work is funded (or part-funded) by the ERDF European Regional Development Fund through the COMPETE Programme (operational programme for competitiveness) and by National Funds through the FCT - Fundação para a Ciência e a Tecnologia (Portuguese Foundation for Science and Technology) within project «FCOMP - 01-0124-FEDER-022701».

Miguel Pinto acknowledge FCT for his $\mathrm{PhD}$ grant (SFRH/BD/60630/2009).

\section{REFERENCES}

[1] Webpage of "SLAM for 3D Map building to be used in a Matching 3D algorithm", available at: "www.fe.up.pt/ dee09013", June, 2012.

[2] J. Borenstain, H. R. Everett, L. Feng and D. Wehe, "Mobile Robot Positioning and Sensors and Techniques", Journal of Robotic Systems, Special Issue on Mobile Robots, Vol. 14 No. 4, pp. 231249.

[3] S. Thrun, W. Burgard, and D. Fox. "Probabilistic Robotics". MIT Press, Cambridge, MA, 2005.

[4] H. Sobreira, A. Paulo Moreira and J. S. Esteves, "Low cost self-localization system with two beacons", Proc. of the 10Th Int. Conf. on Mobile Robots and Competitions (ROBÓTICA 2010), Leiria, Portugal, March 24th, 2010.

[5] M. Pinto, A. P. Moreira and A. Matos, "Localization of Mobile Robots Using an Extended Kalman Filter in a LEGO NXT", IEEE Transactions On Education, 31 May 2012.

[6] M. Lauer, S. Lange and M. Riedmiller, "Calculating the perfect match: an efficient and accurate approach for robot self-localization", RoboCup Symposium, 2005.

[7] J. Minguez, F. Lamiraux, and L. Montesano, "Metric-based scan matching algorithms for mobile robot displacement estimation", IEEE Transactions.

[8] L. Teslić, I. Škrjanc and G. Klančar, "Using a LRF sensor in the Kalman-filtering-based localization of a mobile robot", ISA Transactions (Elsevier), Vol. 49, No. 1, pp. 145-153, January 2010.

[9] G. Grisetti , C. Stachniss and W. Burgard, "Improved Techniques for Grid Mapping with Rao-Blackwellized Particle Filters", IEEE Transactions on Robotics, Vol. 23, No. 1, pp. 34-46, February 2007.

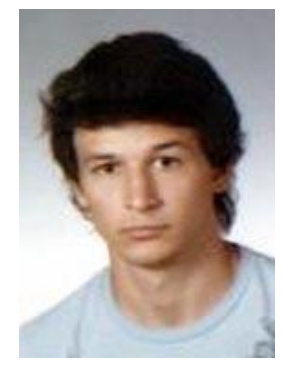

Miguel. Pinto born at Caracas, Venezuela, June 6, 1986, graduated with a M.Sc. degree in Electrical Engineering from the Faculty of Engineering of the University of Porto, Portugal, in 2009.

Since 2009, he has been a Ph.D. student at the Doctoral Programme in Electrical Engineering and Computers (PDEEC), at the Faculty of Engineering of the University of Porto, Portugal. He is a member and develops his research within the Robotic and Intelligent Systems Unit of INESC TEC (the Institute for Systems and Computer Engineering of Porto).

His main research areas are in process control and robotics, navigation and localisation of autonomous vehicles.

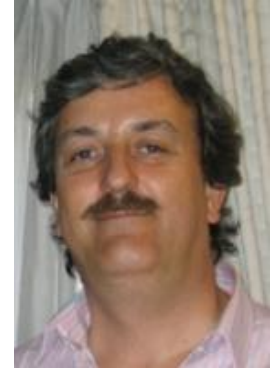

A. Paulo. Moreira born at Porto, Portugal, November 7, 1962, graduated with a degree in Electrical Engineering from the University of Porto in 1986. He then pursued graduate studies at the University of Porto, completing a M.Sc. degree in Electrical Engineering - Systems in 1991 and a Ph.D. degree in Electrical Engineering in 1998. From1986 to 1998 he also worked as an assistant lecturer in the Electrical Engineering Department of the University of Porto. Porto. He is currently a Associated Professor in Electrical Engineering, developing his research within the Robotic and Intelligent Systems Unit of INESC TEC (Unit Coordinator), Porto Portugal. His main research areas are Process Control and Robotics.

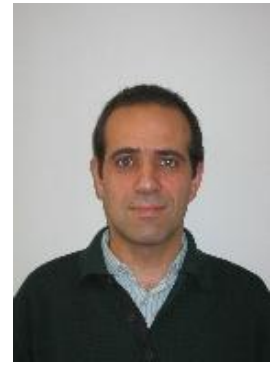

Aníbal. Matos born at Porto, Portugal, completed a B.Sc., a M.Sc. and a Ph.D. degree in Electrical and Computer Engineering at the University Porto in 1991, 1994, and 2001, respectively. He is currently working as an assistant lecturer at the Electrical and Computer Engineering Department of the University of Porto and he is also a researcher at the Robotics and Intelligent Systems Unit at INESC Porto. His research areas include modelling, navigation and control of autonomous vehicles, nonlinear control systems, and marine robotics.

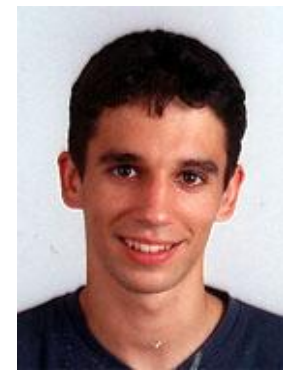

Heber. Sobreira born at Leiria, Portugal, July, 1985, graduated with a M.Sc. degree in Electrical Engineering from the University of Porto in 2009. Since then, he has been a Ph.D. student at Electrical and Computer Engineering Department of the University of Porto, developing his research within the Robotic and Intelligent Systems Unit of INESCPorto (the Institute for Systems and Computer Engineering of Porto). His main research area is navigation and control of indoor autonomous vehicles

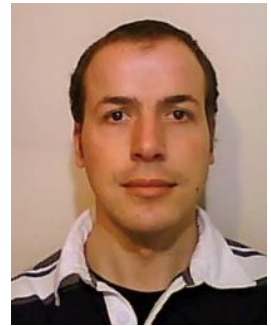

Filipe. Santos born at Porto, Portugal, October 23, 1979, graduated with a M.Sc. degree in Electrical Engineering from the Instituto Superior Técnico (IST) - Lisbon Technical University, Portugal in 2007. Since 2010, he has been a Ph.D. student at the Doctoral Programme in Electrical Engineering and Computers (PDEEC), at the Faculty of Engineering of the University of Porto, Portugal, developing his research within the Robotic and Intelligent Systems Unit of INESC Tec (the Institute for Systems and Computer Engineering of Porto).

His main research areas are in artificial intelligence and robotics, human-robot interface and localisation of autonomous vehicles. 